4. Xu Q,Bao W, Mao R, Pang L Intra-axial tumors of the medullocervical junction: diagnosis and microsurgical treatment. Chin Med J 1998; 111: 968-71.

5. Obón B, Gutiérrez I, Villanueva B, Cía P, Montoiro R, Cárcamo A. Isquemia medular secundaria a hematoma epidural no traumático. An Med Interna (Madrid) 2006; 23: 348-349.

\section{Broncoaspiración oculta de cuerpo extraño}

\section{Sr. Director:}

La broncoaspiración de un cuerpo extraño habitualmente produce un cuadro agudo con disnea, tos intensa, cianosis y estridor, pudiendo ocasionar edema laríngeo, neumotórax, e incluso parada cardíaca. Más rara vez este episodio es de poca intensidad o pasa desapercibido $(1,2)$, quedando el cuerpo extraño broncoaspirado oculto durante meses o años, con clínica subaguda o crónica de tos, expectoración hemoptoica o hemoptisis, fiebre, disnea y dolor torácico, conduciendo frecuentemente a diagnósticos erróneos de asma, enfermedad pulmonar obstructiva crónica (EPOC), neumonía crónica o cáncer de pulmón. Se presenta un caso de broncoaspiración oculta de cuerpo extraño.

Varón, 73 años, con antecedentes de enfermedad cerebrovascular (síndrome vertebro-basilar) antiagregado con ticlopidina (250 mg/día). Exfumador $>20$ paquetes/año desde hacía 10 años; EPOC moderado-grave (FEV1 44\%) en tratamiento con broncodilatadores inhalados (tiotropio y budenosida-formoterol). Neumonía basal derecha hacía 1 año. Cuadro crónico de tos con expectoración amarillenta, ocasionalmente hemoptoica, frecuente opresión torácica y disnea progresiva, así como hiporexia, pérdida de peso y astenia. No fiebre ni febrícula. En analítica destacaban: insuficiencia respiratoria $\left(\mathrm{pO}_{2} 56,7 \mathrm{mmHg}\right)$, anemia $(\mathrm{Hb} 11$ g/dL; Hto 34\%) normocroma normocítica (VCM 81 fL, HCM 26 $\mathrm{pg})$, leucocitosis $(12.400 / \mu \mathrm{L})$ con fórmula normal y velocidad de sedimentación globular (VSG) elevada ( $94 \mathrm{~mm} / 1^{\mathrm{a}}$ hora). Hormonas tiroideas y proteinograma normales. Mantoux y micobacterias en esputo negativos. Electrocardiograma y ecocardiograma sin alteraciones relevantes. En radiografía de tórax se apreciaba pequeño derrame pleural bilateral de predominio derecho, signos de EPOC e hilios aumentados. En tomografía computarizada (TC) torácica helicoidal no había hallazgos de tromboembolismo pulmonar. Se realizó TC torácico y abdominal convencional, con imagen fina y alargada de consistencia ósea dentro del bronquio intermediario derecho (Fig. 1A, flecha), enfisema paraseptal de predominio en vértices, pequeño derrame pleural bilateral y pequeñas adenopatías paratraqueales derechas, subcarinales e hiliares bilaterales. El TC abdominal era normal. Se realizó broncofibroscopia (Fig. 1B), detectándose una placa que ocupaba parcialmente la luz del bronquio intermediario derecho, y una formación mamelonada en zona de contacto de la placa con la pared bronquial (sugestiva de tejido de granulación), que no pudo biopsiarse por ser muy fibrosa. Se extrajo la placa con pinza de biopsia. Se trataba de una estructura ósea aplanada de casi $2 \mathrm{~cm}$. de diámetro máximo (Fig. 1C). Tras la extracción del cuerpo extraño, la evolución posterior fue clínica y radiologicamente favorable. En broncofibroscopia de control a los 3 meses había desaparecido el tejido de granulación en bronquio intermediario derecho. Reinterrogado el paciente, recordaba un episodio de atragantamiento intenso hacía unos 4 años, mientras comía un guiso de conejo.
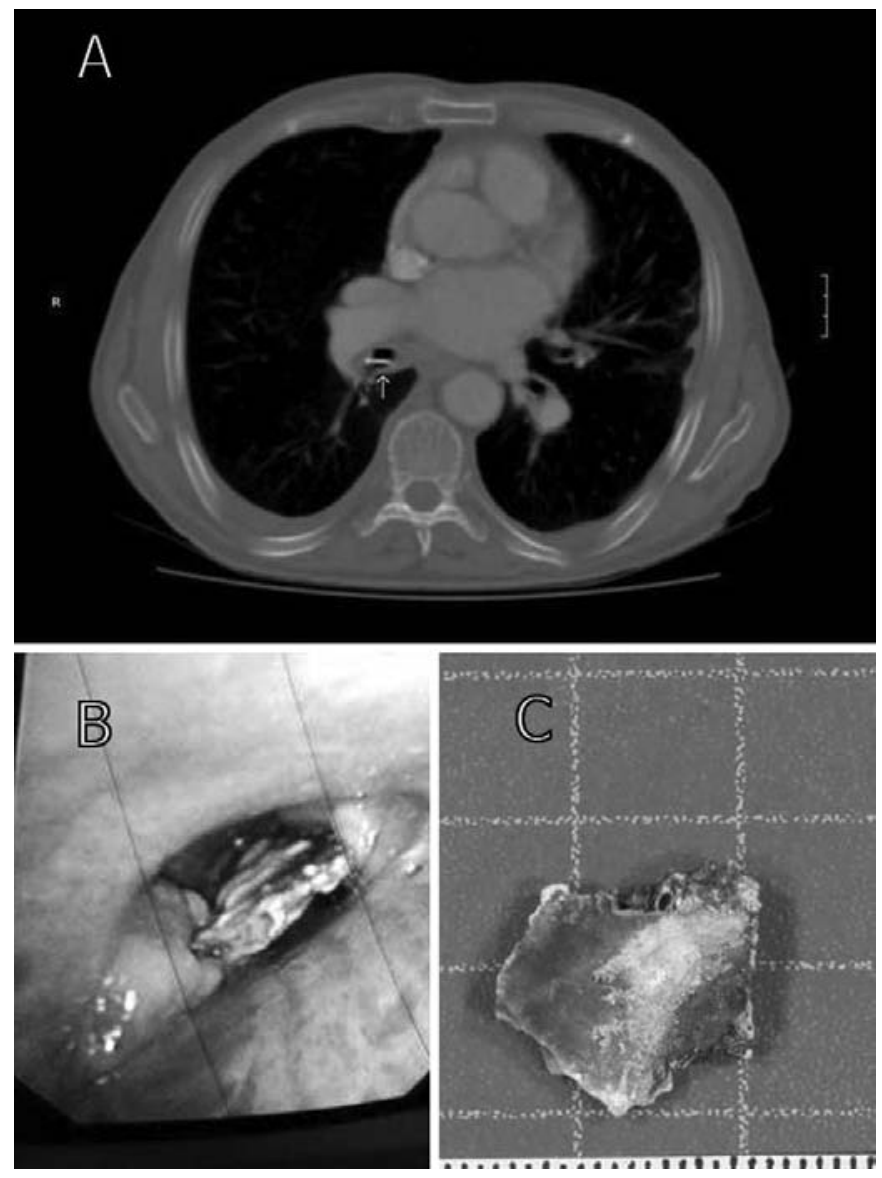

Fig. 1. En C, cada cuadrícula mide $1 \mathrm{~cm}$ de lado.

La broncoaspiración de un cuerpo extraño en adultos (3-5) es menos frecuente que en niños, y habitualmente se produce en pacientes con factores predisponentes tales como: enfermedades neurológicas (Parkinson, epilepsia, retraso mental, parálisis cerebral, enfermedad cerebrovascular, neoplasia cerebral), procedimientos dentales, abuso de alcohol y sedantes, antecedentes de trauma facial e intubación orotraqueal y patología broncopulmonar previa. Es más frecuente en varones y afecta principalmente al árbol bronquial derecho. Los cuerpos extraños broncoaspirados suelen ser partículas de alimentos (huesos, espinas, semillas), comprimidos, piezas dentarias, etc, pudiendo detectarse en radiología simple y/o TC si son metálicos u óseos. Actualmente, la broncofibroscopia es el método diagnóstico más eficaz, y además es un procedimiento terapeútico, permitiendo en muchos casos extraer el cuerpo extraño en la misma exploración diagnóstica (o de forma diferida) mediante el uso de pinzas de biopsia, cesta, asa, electroimán, láser, catéteres (Fogarty, Amplatz) (6), etc. En otros casos para la extracción del cuerpo extraño será preciso utilizar broncoscopia rígida o cirugía (segmentectomía o lobectomía pulmonar) por toracoscopia (7) o toracotomía. Entre las complicaciones crónicas de la broncoaspiración de cuerpos extraños están: atelectasia, neumonía obstructiva, absceso pulmonar y bronquiectasias.

\section{E. Crespo Valadés, A. Blanco Jarava, L. E. Lindo Gutarra,} M. I. García San José ${ }^{1}$

Servicios de Medicina Interna y ${ }^{\prime}$ Neumología. Hospital Virgen de la Salud. Toledo 
1. Yilmaz A, Akkaya E, Damadoglu E, Gungor S. Occult bronquial foreing body aspiration in adults: analysis of four cases. Respirology 2004; 9: 561-3.

2. Zubairi AB, Haque AS, Husain SJ, Khan JA. Foreign body aspiration in adults. Singapore Med J 2006; 47: 415-8.

3. Limper AH, Prakash UBS. Tracheobronchial foreign bodies in adults. Ann Intern Med 1990; 112: 604-9.

4. Chen CH, Lai CL, Tsai TT, Lee YC, Perng RP. Foreign body aspiration into the lower airway in chinese adults. Chest 1997; 112: 129-33.

5. Baharloo F, Veyckermans F, Francis C, Biettlot MP, Rodenstein DO. Tracheobronchial Foreign Bodies: presentation and management in children and adults. Chest 1999; 115: 1357-62.

6. Nalaboff KM, Solis JL, Simon D. Endobronquial foreign body extraction: a new interventional approach. Chest 2001; 120: 1402-05.

7. Wain JC, Mark EJ. Case 33-1997. A 75-Year-Old Man with chest pain, hemoptysis, and pulmonary lesion. N Engl J Med 1997; 337: 1220-6.

\section{Análisis de los 7 primeros años de un programa de trasplante hepático}

\section{Sr. Director:}

Aunque con un cierto retraso con respecto a los primeros trasplantes hepáticos que se llevaron a cabo en EE.UU. durante la década de los años 60 (1), existe en España un número creciente de pacientes trasplantados desde la implantación de programas de trasplante hepático en los hospitales.

Con el propósito de conocer la actividad asistencial de los trasplantes hepáticos $(\mathrm{TH})$ en nuestro hospital, se realiza este análisis de los pacientes que reciben un TH desde el inicio dicho programa en 1998 hasta el año 2005, efectuando una descripción del perfil epidemiológico de los pacientes que precisan TH, estudiando los códigos de inclusión en la lista de espera, las patologías que generaron dichas inclusiones, la compatibilidad isogrupo y la evolución pos-TH (necesidad de reTH y supervivencia posterior).

Durante el periodo de tiempo estudiado, se realizaron un total de 200 TH. El 60\% de los mismos fueron varones cuya media de edad fue de 54 años. Las principales causas por las que precisaron TH fueron la cirrosis enólica $70(35 \%)$, hepatitis vírica 37 $(18,5 \%)$, de origen mixto $38(19 \%)$, fulminante $6(3 \%)$, criptogénica $6(3 \%)$, hepatocarcinoma $4(2 \%)$, cirrosis biliar primaria 2 $(1 \%)$, autoinmune $1(0,5 \%)$, otras causas $10(5 \%) .26$ personas precisaron reTH (13\%). En el momento del TH, 78 pacientes se encontraban en en estadio C de Child (39\%). 183 TH se realizaron de modo programado $(91,5 \%), 10$ se hallaban en urgencia 0 (6 de ellos debido a un fracaso hepático agudo grave), y 7 en urgencia no 0 (urgencia de zona). Los pacientes permanecían en lista de espera una media de 38 días (0-248), que en el caso de urgencia 0 se redujo a 27 horas desde su inclusión. La compatibilidad isogrupo fue del $95 \%$, y el $5 \%$ no era compatible. La supervivencia de los pacientes es del $82 \%$ al año de realización del TH, del $75 \%$ a los 3 años y del $61 \%$ a los 6 años.

Los pacientes que requieren TH son varones, jóvenes, siendo la cirrosis enólica, la hepatitis vírica o de origen mixto las etiologías que más frecuentemente originan su indicación, al igual que en otros estudios en los que el VHC y el hepatocarcinoma son las principales indicaciones (2-4). El período de espera es reducido, sobre todo en el caso de encontrarse en urgencia 0 , ambos tiempos inferiores a las cifras del registro nacional de trasplantes, lo que posiblemente traduce una desigualdad en el acceso a estos programas como ya apuntan algunas investigaciones (3-5). Un elevado porcentaje de pacientes se encuentra en grado muy avanzado de la enfermedad, y una gran proporción de los mismos recibe un hígado de donante compatible. La necesidad de reTH es escasa, motivada fundamentalmente por fallo hepático fulminante al igual que en otros estudios $(6,7)$. La supervivencia al año, así como a los 3 años es elevada, y sobreviviendo más de la mitad de los pacientes una vez transcurridos 6 años. En la actualidad la supervivencia de los pacientes $\mathrm{TH}$ es superior a la del inicio de estos programas, probablemente por una mejoría de las técnicas quirúrgicas y de los cuidados posquirúrgicos en las unidades de cuidados intensivos, así como por el desarrollo de nuevos agentes inmunosupresores, y una mejor selección de los candidatos (2).

\section{B. Obón Azuara, I. Gutiérrez Cía, P. Luque Gómez}

Servicio de Medicina Intensiva. Hospital Clínico Universitario. Zaragoza

1. Starzi TE, Brettschneider L, Penn I, Giles G, Picache R, Putnam CW. Clinical liver transplantation. Transplant Rev 1969; 2: 3-68.

2. Amador A, Charco R, Marti J, Ferrer J, Mans E, et al. Mil trasplantes en el Hospital Clinic I Provincial de Barcelona. Cir Esp 2005; 78: 231-7.

3. Vargas V, Rimola A, Casanovas T, Castells L, Navasa M, Baliellas C, et al. Applicability of liver transplantation in Catalonia at the end of the millennium. A prospective study of adult patient selection for liver transplantation.Transpl Int 2003; 16: 270-5.

4. Cuende Melero N, Miranda Serrano B, Canon Campos JF, Naya Nieto T, Garrido Cantarero G. Criterios de priorización para el acceso al trasplante. El caso del trasplante hepático en España. Med Clin (Barc) 2003; 120: 380-6.

5. Sanchez-Bueno F, Cuende N, Matesanz R, Parrilla P. Emergency organ transplantation in Spain: liver emergency and outcomes. Transplant Proc 2005; 37: 3878-80.

6. Landaverde C, Berenguer M, Aguilera V, San Juan F, Prieto M, Berenguer J. Retrasplante hepático: análisis de los resultados en 50 pacientes. Med Clin (Barc) 2005; 124: 721-5.

7. García Gil FA, Luque P, Ridruejo R, Tome Zelaya E, Guemes A, Serrano MT, et al. Liver transplant, in emergency 0 (UNOS Status 1). Transplant Proc 2006; 38: 2465-7. 